\title{
Raising awareness of oral cancer from a public and health professional perspective
}

\author{
L. M. D. Macpherson ${ }^{1}$
}

\section{Key points}

Discusses the approaches used in an attempt to improve oral cancer awareness through national and community-based campaigns.
Provides an overview of the effectiveness of interventions aimed at raising cancer awareness and promoting early presentation with cancer symptoms. Highlights the limited evidence of long-term effectiveness of such campaigns to date and the need for further work.
Proposes that awareness interventions should be based on longer-term, multi-faceted approaches, acknowledging the social determinants of the disease and health behaviour theory.

Low public awareness of oral cancer and delays in symptomatic patients presenting to health services have been identified as contributing factors to poor survival rates. In order to promote diagnosis and treatment of oral cancer at an earlier stage, public awareness campaigns have been recommended, encouraging those with signs and symptoms to attend primary care services at an earlier stage. This article provides an overview of the evidence of effectiveness of interventions aimed at raising cancer awareness and explores the use of mass media for health behaviour change. The use of awareness campaigns to promote earlier diagnosis of oral cancer is also explored from both a patient and health professional perspective. The findings of the overview suggest that while awareness raising campaigns can increase knowledge of the disease and attendance at health services in the short-term, those at lesser risk often respond, and evidence of longer-term impact is very limited. The translation of knowledge into behaviour change is likely to require a more comprehensive, longer-term, multifaceted approach, acknowledging the social determinants of health and health behaviour theory. More work is required to understand what needs to be included in campaigns to make them effective. Availability and access to appropriately trained and informed primary care personnel is important, particularly for high-risk groups. This is relevant for: supporting those with signs and symptoms to attend services; promoting opportunistic screening; enabling referral of patients to secondary care in a timely manner; and for provision of advice on the major risk factors associated with oral cancer.

\section{Introduction}

In 2012, the reported number of incident cases and deaths from cancer of the lip and oral cavity worldwide were 300,000 and 145,000 , respectively. ${ }^{1}$ The corresponding figures for pharyngeal cancer were 142,000 and 97,000. This paper focuses on cancer of the lip and oral cavity with the term 'oral cancer' being used, as most of the literature relates to these sites and there are additional considerations

IProfessor of Dental Public Health, Dental School, School of Medicine, Dentistry and Nursing, College of MVLS, University of Glasgow

Correspondence to: Lorna Macpherson

Email: Lorna.macpherson@glasgow.ac.uk

Refereed Paper. Accepted 6 August 2018

DOI: $10.1038 /$ sj.bdj.2018.919 concerning the aetiology and vaccine-related preventive approaches associated with oropharyngeal cancer. Oral cancer incidence increases with age and the disease is more common among men. Inequalities exist in oral cancer incidence and mortality, with those from the lower socio-economic groups bearing the greatest burden., ${ }^{2,3}$ The main risk factors for oral cancer are tobacco and alcohol consumption. $^{2}$

While in most countries, the incidence of oral cancer is relatively low in comparison with some other malignancies, it is considered a major public health issue due to low five-year survival rates. These rates are related to a large extent to the often advanced stage of the disease at the time of diagnosis and treatment. Van der Waal ${ }^{4}$ has reported a 5-year survival rate for Stage I oral cancer of approximately
$80 \%$, while the corresponding value for patients with advanced disease (Stages III/IV) is approximately $20 \%$.

Approximately $50 \%$ of patients with oral cancer worldwide present with advanced disease. ${ }^{4}$ Furthermore, Douglas and co-workers $^{5}$ have shown that, in a group of Scottish patients, the number of symptoms a patient presents with is significant for both 5-year and 12-year survival, after adjustment for stage and age.

More advanced staging of disease at diagnosis and treatment can also result in significant functional impairment as well as disfigurement, with consequent impact on the quality of life of patients.

Thus, it is advocated that efforts should be made to reduce the morbidity and mortality from oral cancer through lesion detection at 
a less advanced stage. Potential ways of diagnosing oral cancer at an earlier stage include population screening of high risk groups; opportunistic screening by primary healthcare professionals; and reduction in delays by both patients and primary healthcare professionals from the first sign or symptom to establishment of a definitive diagnosis. ${ }^{6}$ While the first approach was found to be effective in high-risk individuals in a high prevalence population in India, ${ }^{7}$ it is less effective in countries where the overall prevalence is relatively low. Therefore, efforts tend to focus on raising awareness of oral cancer among the population and promoting access to primary care services if symptoms persist for more than a few weeks. Encouraging regular attendance at dental practices is also advocated to enable opportunistic screening to be undertaken. Furthermore, the dental and wider primary healthcare team should be appropriately trained to respond to symptomatic patients and to conduct opportunistic screening for oral cancer.

\section{Public awareness of oral cancer}

As oral cancer is relatively rare in many countries, it is perhaps not surprising that symptom recognition and awareness of risk factors is limited. ${ }^{8}$ Studies conducted in the UK, Western Europe, the USA and Australia all highlight a relatively low level of public awareness of oral cancer, its signs and symptoms and risk factors. ${ }^{9-13}$ This is particularly the case for population groups (lower SES, education levels and health literacy) most likely to be affected. ${ }^{11-13}$

Furthermore, a qualitative study, conducted in Scotland, explored the experiences of young oral cancer patients ${ }^{14}$ and confirmed gaps in understanding and awareness of oral cancer. Although many patients had heard of mouth cancer, they did not think their symptoms were indicative of the disease. Consequently, many initially self-managed their symptoms and a culture of not wanting to bother the medical/dental practitioner was identified for something not perceived to be serious. Similar findings were reported from a telephone survey of oral cancer patients in the Liverpool area. ${ }^{15}$ Other reasons given for patients' delay include fear of a diagnosis of cancer and limited access to primary healthcare professionals. ${ }^{4}$

The association between tobacco use and oral cancer is recognised by many individuals, but much lower numbers are aware of alcohol consumption as a risk factor. ${ }^{13,16}$
The consensus and recommendations made in all the studies outlined above has been that more needs to be done to raise public awareness of the signs and symptoms of oral cancer and its risk factors, with most advocating the use of public awareness campaigns.

\section{Promotion of cancer awareness}

Today there are many annual campaigns organised by international and national bodies to raise public awareness of cancer and other health matters. Examples include World Cancer Day, World AIDS Day, World Oral Health Day and Mouth Cancer Action Month in the UK. Many programmes and events are organised at the local level to support such campaigns. Additionally, a plethora of smaller, individual and community-based interventions have been used to raise awareness of cancer, using a variety of approaches and methodologies.

In 2009 , a systematic review was published ${ }^{17}$ which examined the evidence of the effectiveness of interventions aimed at raising cancer awareness and promoting early presentation with cancer symptoms. The authors found some limited evidence that interventions delivered at the individual level could promote cancer knowledge and awareness in the short term, particularly for more intensive and tailored interventions, but no evidence that this translated into promoting early presentation with cancer symptoms. However, many of the studies included in the review focused only on short-term knowledge outcomes. The individual level interventions included written information via letters, brochures and leaflets; telephone counselling; and computer interactive programmes within the primary care setting.

In relation to community-based interventions, the review found that approaches such as small group education programmes and health promotion programmes in leisure centres, workplaces and health clubs were again associated with limited evidence of effectiveness in promoting cancer awareness. There was some evidence that campaigns such as Breast Cancer Awareness Month in the USA and interventions by community-based health advocates could be associated with diagnosis of breast cancer at an earlier stage. Similar findings were reported in relation to programmes associated with malignant melanoma. However, the authors of the review cautioned against attributing the down-staging of cancers to the effect of raising public awareness alone. They concluded that more work was required to understand what needs to be included in a campaign to make it effective and cost-effective.

\section{Mass media campaigns for health behaviour change}

A review of the use of mass media campaigns to change health behaviour was published in the Lancet in $2010 .^{18}$ It concluded that it was possible for mass media campaigns to effect positive changes in health-related behaviours across large populations. The likelihood of success was assessed to be substantially increased by the application of multiple interventions and when the target behaviour was one-off or episodic (eg, cancer screening) rather than on-going. The concurrent availability of and access to key services were recognised as crucial to enable individuals motivated by such campaigns to act on them.

Additionally, approaches that shape the treatment of a public health issue by news and entertainment media were also thought to represent a promising strategy to complement conventional mass media approaches. This concurs with the views of Verma and coworkers, ${ }^{19}$ who recommended that engaging makers of popular television programmes in a health promotion agenda may be a useful methodology for shaping behaviour norms and promoting health behaviours. The impact on screening uptake following news of a celebrity's cancer diagnosis has also received attention. An example of this would be the so-called 'Jade Goody effect' in the UK. ${ }^{20}$ However, sustainability of impact is usually an issue. Many people now also turn to the internet for health information and this can be a useful mechanism for providing up-to-date and relevant cancer awareness messages to the public. ${ }^{21}$

In relation to mass media campaigns associated with cancer risk factors such as tobacco and alcohol, Wakefield and co-authors recognised that commercial marketing, social norms and addictions can present barriers, meaning that positive outcomes are often difficult to maintain. However, a recent Cochrane review ${ }^{22}$ investigating mass media interventions for smoking cessation in adults concluded there was evidence that comprehensive programmes that included mass media campaigns can be effective in changing smoking behaviours of adults. The intensity and duration of such tobacco control programmes were considered relevant to effectiveness. The authors acknowledged, however, that the variable methodological quality of 
studies included in the review, short length of follow-ups and secular trends made quantification of the extent of the effectiveness of such programmes difficult.

A systematic review on the effectiveness of mass media campaigns to reduce alcohol consumption and harm has also recently been published. ${ }^{23}$ The authors concluded that such campaigns are often recalled by members of the public and can change knowledge, attitudes and beliefs about alcohol. However, they found very little evidence of an impact on alcohol consumption.

For public awareness campaigns it is important to consider the target audience and one of the issues associated with a mass media approach is its reach and impact on different sections of society. Often messages are acted upon most by those with higher socioeconomic status, education, and resources, while those from more disadvantaged circumstances find it much more challenging to implement behaviour change. ${ }^{24}$ This is a particular issue for oral cancer in terms of dental attendance and risk factor behaviours, given the strong associations with low socioeconomic circumstances. ${ }^{2,25}$

According to the Scottish Government Ministerial Task Force on health inequalities, ${ }^{24}$ characteristics of interventions that are less likely to be effective in reducing health inequalities include:

- Information-based campaigns (mass-media information campaigns)

- Written materials (pamphlets, food labelling)

- Campaigns reliant on people taking the initiative to opt in

- Campaigns/messages designed for the whole population

- Approaches which involve significant price or other barriers.

The review of mass media campaigns ${ }^{18}$ produced policy recommendations for governments, practitioners and professional bodies to review when considering such campaigns and these are as follows:

- Mass media campaigns should be included as key components of comprehensive approaches to improving population health behaviours

- Sufficient funding must be secured to enable frequent and widespread exposure to campaign messages continuously over time, especially for ongoing behaviours

- Adequate access to promoted services and products must be ensured
- Changes in health behaviour might be maximised by complementary policy decisions that support opportunities to change, provide disincentives for not changing, and challenge or restrict competing marketing

- Campaign messages should be based on sound research of the target group and should be tested during campaign development

- Outcomes should undergo rigorous independent assessment and peer-reviewed publication should be sought.

\section{Promotion of oral cancer awareness}

Promotion of oral cancer awareness can involve population-based programmes, multi-faceted mass media approaches, community-based initiatives and one-to-one interventions.

In South East Asia, where the prevalence of oral cancer is high, many programmes have been developed in an attempt to raise awareness of the disease and its risk factors. Campaigns in India include screening of high risk individuals, encouraging oral self-examination, and involvement of village health workers in promoting early detection and awareness of head and neck cancers. ${ }^{7,26,27}$

\section{Annual national awareness campaigns}

In other parts of the world, other approaches are used and a number of countries have introduced national mouth cancer awareness days or weeks on an annual basis. ${ }^{28-30}$ These aim to engage with the public and primary healthcare professionals and use a variety of methodologies. The programmes often give facts and figures about the disease, highlight signs, symptoms and risk factors and provide advice on self-examination and on visiting dental or medical practitioners if problems persist for more than a few weeks. Local activities across the countries are encouraged, with media involvement and, in many cases, increased opportunities provided for a free mouth examination.

Short-term evaluation of such programmes suggests they can result in increased awareness of oral cancer, increased activity at secondary care centres and that they may pick up some malignant lesions. ${ }^{31,32}$ Evidence on the longerterm impacts of such campaigns is, however, very limited. While the main aim of such programmes is to reach groups at higher risk of oral cancer and who are less likely to visit a dentist on a regular basis, it is acknowledged that it is often the 'worried well' who respond in greater numbers to such programmes ${ }^{31}$ and that many people without relevant lesions are seen. The cost and opportunity costs to health services of such initiatives therefore need to be considered. Careful thought needs to go into the planning, theoretical behavioural underpinning, and content of such national programmes. The importance of also encouraging those at greater oral cancer risk to attend for opportunistic screening as part of a regular dental check-up is evident. This can be important for early detection as it has been stated that premalignant and early oral cancer lesions are often asymptomatic, with no pain or discomfort felt by patients. ${ }^{33}$

\section{Multi-component, theory-based one- off programmes}

In addition to the one to four week annual campaigns, other one-off mass media programmes have been used to promote increased awareness of oral cancer and encourage early detection via engagement with primary care services. An example is the West of Scotland Cancer Awareness Project. ${ }^{34}$ This was the first campaign of its type in the UK, bringing together five regional health boards with a combined population of over 2.5 million. The programme targeted an at-risk population of adults over 45 years of age from lower socio-economic groups. It ran over a six month period. The campaign was developed using social cognitive theory and was grounded in social marketing with much involvement of the target group and other stakeholders during programme development. ${ }^{35}$ The main features of this National Lottery-funded programme involved targeted advertisements on television and radio; posters; leaflets; and direct mail drops in key target communities. There was also generation of news stories for broadcast by local and national media and training was provided for primary care medical and dental practitioners and community pharmacists across the region. Secondary care clinicians were also involved from the outset to ensure that referral and clinical systems were prepared for any increased demand.

An evaluation of the programme, which included interviews at baseline and up to 12 months following the campaign, suggested it had been successful in raising awareness of oral cancer and its symptoms and in encouraging individuals to consider consulting primary care health professionals should symptoms be experienced. ${ }^{35}$ Additionally, many attending secondary care rapid access clinics during 
the campaign were from the target group, with good awareness of the campaign among these patients. However, while some referrals included expected lesion types, there was also a high proportion of non-urgent lesions. ${ }^{34}$ Issues associated with campaign sustainability and maintenance of awareness levels without causing message fatigue were also highlighted. ${ }^{35}$

An oral cancer marketing campaign in Michigan, ${ }^{36}$ targeted particularly at AfricanAmerican men, worked with local communities to produce culturally-appropriate materials. Features of the public campaign included numerous radio advertisements, billboard displays and multiple educational sessions with community groups in settings including churches and homeless shelters. Educational programmes were also provided to dentists and physicians in the area as the aim of the campaign was to encourage the public to ask for screening for oral cancer and persuade providers to offer screening to their patients. The programme resulted in more requests for screening from patients and some increase in screening activity occurred during the campaign period. However, the authors acknowledged it was not clear whether patients most at risk of developing oral cancer were responsible for the increase in numbers requesting screening. They also recognised that behaviour change may require more intensive and long-term programmes and stressed the importance of ensuring such campaigns are focused and integrated within existing health systems.

More recently, the outcomes from the first phase of developing an oral cancer awareness campaign in Germany have been published. ${ }^{37}$ The comprehensive, multi-component programme, targeted at those with higher risk status, is based on the transtheoretical model of behaviour change. Robust process evaluation is being undertaken to ensure that uptake of key messages occurs among the target population, before moving onto the development of interventions to address attitude and motivation, the next stages deemed necessary for sustained health behaviour change. ${ }^{37}$

\section{Local community-based programmes}

Many smaller and more localised educationbased oral cancer awareness campaigns have been undertaken worldwide, including within the UK. The consensus is that local programmes, using materials such as posters and leaflets, tend at most to result in increased awareness in the short-term, and that the impacts of such low-intensity campaigns are usually of short duration, ${ }^{38}$ with limited if any behaviour change. Furthermore, such an approach can result in most knowledge gain among those at lesser risk of the disease. This concurs with the inequalities literature. ${ }^{24}$ The need to develop campaigns with input from target communities is recognised. For example, the importance of development of culturally sensitive approaches within particular high-risk ethnic minority communities has been highlighted. ${ }^{38,39}$

Community-based health advocates, using a more active approach than a simple messagebased programme, have been shown to have some effect in raising cancer awareness and promoting presentation at screening. ${ }^{17}$ Village health workers have been utilised in this way in India in relation to oral cancer. ${ }^{27}$ In Scotland, the national child oral health improvement programme utilises community-based dental health support workers, particularly in more disadvantaged areas, to raise awareness of oral health and encourage regular dental attendance. ${ }^{40}$ This targeted intervention, tailored to the needs of individual families, has been shown to be effective in promoting access to dental services. ${ }^{41}$ Close working with community groups, services and agencies is also encouraged to promote an inter-sectoral style of local partnership working to raise awareness, provide practical support and enable behaviour change among residents. A similar approach, utilising primary care community link workers ${ }^{42}$ may be helpful to raise awareness of oral cancer and help facilitate behaviour change and attendance at dental primary care within targeted communities.

Overall, it is recognised that further work is required to identify appropriate approaches for raising awareness of oral cancer and its risk factors in high-risk communities. ${ }^{43}$ Ford and Farah $^{44}$ highlight the importance of recognising the structural and psychosocial factors involved. They suggest that more work is required to understand the barriers to patients attending health services following recognition of symptoms. Such knowledge could help inform future campaign activity. They cite cognitive and emotional responses together with beliefs as factors influencing the initial decision to seek help. Once this decision is made, other issues such as dental anxiety, cost and ease of access to healthcare can impact on the ease of translating this decision into actual attendance.

\section{Role of the primary healthcare team}

Although high-risk groups are less likely to attend primary care dental services on a regular basis, the dental team provides an important resource for increasing awareness of oral cancer and advising on risk factors on an individual basis, particularly for those with risk behaviours. One-to-one health advice, given to patients at the chairside, can be effective if tailored to individual needs and circumstances. The dental team also has an important role in the opportunistic screening for oral cancer in all patients seen.

When patients become aware of signs and symptoms associated with oral cancer, they may well present to a general medical practitioner rather than a dentist. For example, findings from the West of Scotland oral cancer awareness campaign showed that more patients referred to secondary care services first presented to their doctor (59\%) rather than their dentist (29\%). ${ }^{34}$ The wider primary healthcare team therefore needs to be equipped to respond to patients presenting with signs and symptoms of the disease. This includes the referral of appropriate cases to secondary care services in a timely manner.

Ford and Farah have indicated that many of those at most risk of oral cancer are unlikely to be regular dental attenders and that this reduces the effectiveness of opportunistic screening within primary dental care. ${ }^{44}$ Such patients, particularly smokers and heavy drinkers, are, however, more likely to access primary medical care. The authors therefore suggest that the primary medical team can play a role in opportunistic screening for oral cancer in this patient group. Additionally, community link-workers attached to medical practices can help to facilitate attendance at dental primary care services.

A number of studies have investigated oral cancer awareness among primary care doctors, dentists and community pharmacists and have recommended the need for further training in relation to detection of lesions and patient referral pathways. ${ }^{45,46}$ Oral cancer awareness campaigns often include initiatives aimed at health professionals within their wider programmes. However, such programmes can still result in a high number of inappropriate referrals with consequent impact on secondary care services. ${ }^{34}$ This again reinforces the need for appropriate training of primary care practitioners during such campaigns. Additionally, 
some regulators, such as the General Dental Council, advise regular training of the clinical dental team on this topic, and evidence-based guidelines and online training packages are available in many countries.

\section{Promoting risk factor behaviour change}

The literature highlights the difficulties in effecting improved population health outcomes from awareness campaigns aimed solely at the early diagnosis and treatment of cancer. Therefore, in addition to secondary prevention, the importance of including primary prevention in oral cancer related programmes is evident. For tobacco and alcohol control activity, a common-risk factor approach is advocated, as part of a non-communicable disease strategy. In addition to tailored one-to-one advice, more upstream community-based and policy initiatives (eg fiscal measures, smoke-free areas) should also form part of such strategies.

\section{Conclusions}

Efforts to improve the detection of oral cancer at an earlier stage should focus on both patients and primary care practitioners and need to be based on an understanding of the structural and social determinants of the disease. ${ }^{44}$

Delay in patients presenting to health services is considered a major factor responsible for late diagnosis and poor oral cancer outcomes. ${ }^{8}$ Consequently, population-based oral cancer awareness campaigns have been established in many countries. While they can provide a useful function in improving knowledge and motivation, longer-term impacts associated with increased service access and diagnosis of lesions at an earlier stage have yet to be seen. Additionally, without targeting, such programmes may have a lesser effect on those at highest risk.

Multi-component, theory-based campaigns, targeted at high-risk groups, may have greater potential for translating knowledge gained into behaviour change. However, sustainability is again an issue. The generic cancer awareness literature acknowledges that more work is required to understand what needs to be included in public awareness campaigns to promote early cancer detection and make programme outcomes sustainable and cost-effective. ${ }^{17}$

The short-term nature of campaign messaging often does not take into account the wider social determinants of health or address the cognitive, emotional and attitudinal factors that can influence translation of information to behaviour. ${ }^{44}$ Future activity needs to acknowledge determinants that influence health behaviours, have appropriate theoretical behavioural underpinning and be evaluated rigorously using appropriate study designs.

The provision of more sustained support and advice in communities with a higher oral cancer risk is as important as the high profile campaigns. Inter-sectoral working among community groups, agencies and workers can help raise awareness and provide appropriate support on issues relating to oral cancer, including risk factors.

The availability of primary care services is crucial to allow individuals targeted and motivated by oral cancer awareness campaigns to act on them. Appropriately informed and trained healthcare teams are essential to reduce delays between patient presentation and referral to secondary care. Additionally, opportunistic screening for oral cancer can be carried out as part of a routine dental visit. The role of the wider primary healthcare team in opportunistic screening of high risk groups who are less likely to attend dental practice could also be explored.

Finally, as survival rates remain poor and the limitations of current cancer awareness campaigns are evident, the importance of primary prevention is clear. Consequently, programmes related to oral cancer also need to focus on tobacco and alcohol control as part of a multi-faceted, common-risk factor and noncommunicable disease agenda.

\section{Acknowledgement}

Thanks are due to Professor David Conway for providing constructive comments on the manuscript before submission.

1. Ferlay J, Soerjomataram I, Dikshit R et al. Cancer incidence and mortality worldwide: sources, methods and major patterns in GLOBOCAN 2012. Int I Cancer 2015; 136: E359-E386.

2. Conway D I, Brenner D R, McMahon A D et al. Estimating and explaining the effect of education and income on head and neck cancer risk: INHANCE consortium pooled analysis of 31 case-control studies from 27 countries. Int I Cancer 2015; 136: 1125-1139.

3. Macmillan Cancer Support and Information Services Division (ISD). Head and Neck Cancer Survival and Deprivation. Macmillan-ISD Cancer Pathways, Edinburgh, 2017. Available at https://www.macmillan.org.uk/ about-us/what-we-do/evidence/research-funding/ourpartnerships/information-services-division-scotland. html (accessed June 2018).

4. van der Waal I. Are we able to reduce the mortality and morbidity of oral cancer; some considerations. Med Oral Patol Cir Bucal 2013; 18: e33-e37.

5. Douglas C M, Ingarfield K, McMahon A D, Savage S A, Conway D I, MacKenzie K. Presenting symptoms and long-term survival in head and neck cancer. Clinical Otolaryngology 2018; 43: 795-804.
6. Gomez I, Warnakulasuriya S, Varela-Centelles P I et al. Is early diagnosis of oral cancer a feasible objective? Who is to blame for diagnostic delay? Oral Dis 2010; 16 333-342.

7. Sankaranarayanan R, Ramadas K, Thomas $\mathrm{G}$ et al. Effect of screening on oral cancer mortality in Kerala, India: A cluster-randomised controlled trial. Lancet 2005; 365: 1927-1933.

8. van der Waal I, de Bree R, Brakenhoff R, Coebergh J-W. Early diagnosis in primary oral cancer: is it possible? Med Oral Patol Cir Bucal 2011; 16: e300-e305.

9. Hertrampf K, Wenz H-J, Koller M, Wiltfang J. Public awareness about prevention and early detection of oral cancer: a population-based study in Northern Germany. J Craniomaxillofac Surg 2012; 40: e82-e86.

10. Luryi A L, Yarbrough W G, Niccolai L M et al. Public awareness of head and neck cancers. A cross-sectional survey. JAMA Otolaryngol Head Neck Surg 2014; 140: 639-646.

11. Logan $\mathrm{H}$. Public awareness of oral and pharyngeal cancer: what can a dentist do? Todays FDA 2014; 26: 38-41.

12. Formosa J, Jenner R, Nguyen-Thi M-D, Stephens C, Wilson C, Ariyawardana A. Awareness and knowledge of oral cancer and potentially malignant oral disorders among dental patients in far north Queensland, Australia. Asian Pac J Cancer Prev 2015; 16: 4429-4434.

13. Monteiro L S, Warnakulasuriya S, Cadilhe $S$ et al. Oral cancer awareness and knowledge among residents in the Oporto city, Portugal. J Investig Clin Dent 2016; 7: 294-303.

14. Grant E, Silver K, Bauld L, Day R, Warnakulasuriya S. The experiences of young oral cancer patients in Scotland: symptom recognition and delays in seeking professional help. Br Dent J 2010; 208: 465-471.

15. Rogers $S \mathrm{~N}$, Hunter R, Lowe $D$. Awareness of oral cancer in the Mersey region. Br J Oral Maxillofac Surg 2011; 49: $176-181$.

16. Posorski E, Boyd L, Giblin L J, Welch L. Oral cancer awareness among community-dwelling senior citizens in Illinois. J Community Health 2014; 39: 1109-1116.

17. Austoker J, Bankhead C, Forbes L J L et al. Interventions to promote cancer awareness and early presentation: systematic review. Br J Cancer 2009; 101: S31-S39.

18. Wakefield M A, Loken B, Hornik R C. Use of mass media campaigns to change health behaviour. Lancet 2010; 376: 1261-1271.

19. Verma T, Adams J, White M. Portrayal of health-related behaviours in popular UK television soap operas. J Epidemiol Community Health 2007; 61: 575-577.

20. Casey G M, Morris B, Burnell M, Parberry A, Singh N Rosenthal A N. Celebrities and screening: a measurable impact on high-grade cervical neoplasia diagnosis from the 'Jade Goody effect' in the UK. Br J Cancer 2013; 109: 1192-1197.

21. Cancer Research UK. Mouth and oropharyngeal cancer Available at https://about-cancer.cancerresearchuk.org/ about-cancer/mouth-cancer (accessed June 2018).

22. Bala M M, Strzeszynski L, Topor-Madry R. Mass media interventions for smoking cessation in adults. Cochrane Database Syst Rev 2017; DOI: 10.1002/14651858. CD004704.pub4.

23. Young B, Lewis S, Katikireddi S V et al. Effectiveness of mass media campaigns to reduce alcohol consumption and harm: a systematic review. Alcohol Alcohol 2018; 53: 302-316.

24. Macintyre S. Inequalities in health in Scotland: what are they and what can we do about them? Glasgow: MRC Social \& Public Health Sciences Unit, 2007. Available at www.sphsu.mrc.ac.uk/reports/OP017.pdf (accessed June 2018).

25. Purkayastha M, McMahon A D, Gibson J, Conway D I. Trends of oral cavity, oropharyngeal and laryngeal cancer incidence in Scotland (1975-2012) - A socioeconomic perspective. Oral Oncol 2016; 61: 70-75.

26. Singh K, Sharma D, Kaur M, Gauba K, Thakur J S, Kumar R. Effect of health education on awareness about oral cancer and oral self-examination. J Edu Health Promot 2017; 6: 27.

27. Mishra G S, Bhatt S H. Novel programme of using village health workers in early detection and awareness of head and neck cancers: audit of a community screening 
programme. Indian J Otolaryngol Head Neck Surg 2017; 69: 488-493.

28. Oral Health Foundation. Mouth Cancer Action Month Available at https://www.dentalhealth.org/Pages/Category/mouth-cancer-action-month (accessed June 2018).

29. The Oral Cancer Foundation. Oral Cancer Awareness Month. Available at https://oralcancerfoundation.org/ april-oral-cancer-awareness-month/ (accessed June 2018).

30. Awareness Days Australia. Mouth Cancer Action Month 2018. Available at https://www.awarenessdays.com/ au/awareness-days-calendar/mouth-cancer-actionmonth-2018-3/ (accessed June 2018).

31. MacCarthy D, Nunn J, Healy C M et al. Outcomes from the first mouth cancer awareness and clinical check-up day in the Dublin Dental University Hospital. J Ir Dent Assoc 2012; 58: 101-108.

32. Rafiq R, Brocklehurst P, Rogers S N. Effect of mouth cancer awareness week on urgent suspected head and neck cancer referrals. Br J Oral Maxillofac Surg 2013; 51: e183-e185.

33. Poh C F. Improving public awareness and outcomes for oral cancer. Future Sci OA 2016; 2: FS0103.
34. Rodgers J, Macpherson L M D, Smith G L F, Crighton A J, Carton A T M, Conway D I. Characteristics of patients attending rapid access clinics during the West of Scotland Cancer Awareness Programme oral cancer campaign. Br Dent J 2007; 202: E28.

35. Eadie D, Mackintosh A M, MacAskill S, Brown A. Development and evaluation of an early detection intervention for mouth cancer using a mass media approach. $\mathrm{Br}$ J Cancer 2009; 101: S73-S79.

36. Ismail A I, Jedele J M, Lim S, Tellez M. A marketing campaign to promote screening for oral cancer. JADA 2012; 143: e57-e66.

37. Baumann E, Koller M, Wiltfang J, Wenz H-J, Moller $B$, Hertrampf K. Challenges of early detection of ora cancer: raising awareness as a first step to successful campaigning. Health Educ Res 2016; 31: 136-145.

38. Croucher R, Islam S S, Nunn H. Campaign awareness and oral cancer knowledge in UK resident adult Bangladeshi: a cross-sectional study. Br J Cancer 2011; 105: 925-930.

39. Siddique I, Mitchell D A. The impact of a communitybased health education programme on oral cancer risk factor awareness among a Gujarati community. Br Dent $J$ 2013; 215: E7.
40. Macpherson L M D, Ball G E, Brewster L et al. Childsmile: the national child oral health improvement programme in Scotland. Part 1: establishment and development. $\mathrm{Br}$ Dent J 2010; 209: 73-78.

41. Hodgins F, Conway D, Gnich W, Macpherson L, Sherriff A. Childsmile dental health support workers impact on child dental participation in Scotland. European Association of Dental Public Health, Budapest, Hungary, 2016.

42. Scottish Government. Fairer Scotland Action Plan 2016. Available at https://www.gov.scot/ Resource/0050/00506841.pdf (accessed June 2018).

43. Williams M, Bethea J. Patient awareness of oral cancer health advice in a dental access centre: a mixed methods study. Br Dent J 2011; 210: E9.

44. Ford P J, Farah C S. Early detection and diagnosis of oral cancer: strategies for improvement. J Cancer Pol 2013; 1: e2-e7.

45. Carter L M, Ogden G R. Oral cancer awareness of general medical and general dental practitioners. Br Dent J 2007; 203: E10.

46. Rogers S N, Lowe D, Catleugh M, Edwards D. An oral cancer awareness intervention in community pharmacy. Br J Oral Maxillofac Surg 2010; 48: 498-502. 